\title{
COVID-19 Pandemic, Disparity and Vaccine: An Update
}

\author{
Prabir Manda ${ }^{1 *}$ and Anita Mandal ${ }^{2}$ \\ ${ }^{\mathbf{1} B i o l o g y}$ Program, Department of Mathematics \& Sciences, Edward Waters College, Jacksonville, FL 32209. \\ ${ }^{2}$ Department of General Studies, Edward Waters College, 1658 Kings Road, Jacksonville, FL 32209 \\ *Corresponding author: Dr. Prabir K Mandal, Ph.D., Professor\& Chair, Department of Mathematics \& Sciences, Edward Waters College, \\ Jacksonville, FL 32209. \\ Received date: December 15, 2020; Accepted date: December 23, 2020; Published date: February 01,2021 \\ Citation: Anita Mandal and Prabir K. Mandal, (2021) COVID-19 Pandemic, Disparity and Vaccine: An Update. J, Biotechnology and \\ Bioprocessing 2(1); DOI: 10.31579/2766-2314/020
}

Copyright: ( ) 2021, Prabir K. Mandal. This is an open access article distributed under the Creative Commons Attribution License, which permits unrestricted use, distribution, and reproduction in any medium, provided the original work is properly cited.

\begin{abstract}
Coronaviruses are a diverse group of viruses infecting many different animals, and they can cause mild to severe respiratory infections in humans. In 2002 and 2012, respectively, two highly pathogenic coronaviruses with zoonotic origin, severe acute respiratory syndrome coronavirus (SARS-CoV) and Middle East respiratory syndrome coronavirus (MERS-CoV), emerged in humans and caused fatal respiratory illness, making emerging coronaviruses a new public health concern in the twenty-first century1. In early December 2019, an outbreak of coronavirus disease 2019 (COVID-19), caused by a novel severe acute respiratory syndrome coronavirus 2 (SARS-CoV-2), occurred in Wuhan City, Hubei Province, China. On January 30, 2020 the World Health Organization declared the outbreak as a Public Health Emergency of International Concern. As of February 14, 2020, 49,053 laboratory-confirmed and 1,381 deaths have been reported globally.
\end{abstract}

Keywords: covid-19, disparity, epidemic, SARS-CoV2, vaccine

\section{Introduction}

COVID- 19 better known as the corona virus is an infectious disease that is transmitted primarily in close contact with another person or exchange of body fluids or saliva through sneezing, talking and coughing. The droplets can land on another person's mouth, nose, hand and even sometimes when they inhale in their lungs droplets can also land on the surface of different objects and areas that most people touch or interact with which is why more pre-cautionary actions are being taken into account. This virus caused a nationwide pandemic which in turn caused a global shut down where no one was permitted to enter or leave. Traveling was also prohibited and cities were on lockdown and people were not allowed to leave their homes. With the virus constantly changing new symptoms arising, the need for new preventative strategies is now more needed than ever due to this respiratory illness. It is vital to understand that with rapid and constant changes within the virus information regarding risk factors are limited and this virus is fairly new to everyone. In some cases people who are infected with the disease have respiratory problems and can become seriously ill especially people with prior respiratory problems are at a higher risk and eventually the severity of the disease leads to death. Additionally, those of all ages with underlying medical conditions typically older people as overtime their organ systems function differently and begin to shut down. People with heart disease, diabetes, or lung disease appear to be at higher risk in developing severe COVID-19 compared to those without these conditions. As more data become available, additional risk factors for severe the disease may be identified. There are a number of ways to prevent the spread of COVID19 infection; avoid touch eyes, nose and mouth, avoid close contact with people who are sick, stay home if you are sick, when coughing or sneezing use a tissue and then dispose it properly, wear a mask and remain a six feet distancing when going out, clean and disinfect frequently touched surfaces and items, and when washing your hands use hand soap and wash thoroughly or use alcohol based sanitizer. Long-standing systemic health and social inequities have put many people from racial and ethnic minority groups at increased risk of getting sick and dying from COVID19. The term "racial and ethnic minority groups" includes people of color with a wide variety of backgrounds and experiences. But some experiences are common to many people within these groups, and social determinants of health have historically prevented them from having fair opportunities for economic, physical, and emotional health [2]. The COVID-19 pandemic may change some of the ways we connect and support each other.

The World Health Organization announced an official name for the disease that is causing the 2019 novel coronavirus outbreak, first identified in Wuhan, China. The new name of this disease is coronavirus disease 2019, abbreviated as COVID-19. In COVID-19, 'CO' stands for 'corona,' 'VI' for 'virus,' and 'D' for disease. Formerly, this disease was referred to as "2019 novel coronavirus" or "2019-nCoV [2]. In December 2019, a new highly transmitted coronavirus, which was provisionally named 2019-novel coronavirus, was spread in Wuhan, China [3]. Currently, more than 73,580,947 cases, and more than 1,636,741 deaths have been reported globally. This new highly transmitted coronavirus is responsible for the development of severe acute respiratory distress syndrome. Due to this disorder, a great number of patients are hospitalized in the intensive care unit followed by connection to extracorporeal 
membrane oxygenation for breath supporting and survival [3]. The COVID-19 outbreak has also been associated with severe economic impacts globally due to the sudden interruption of global trade and supply chains that forced multinational companies to make decisions that led to significant economic losses. COVID-19 spread is rapid, including imported cases in travelers, cases among close contacts of known infected individuals, and community-acquired cases without a readily identifiable source of infection. Critical shortages of personal protective equipment and ventilators are compounding the stress on overburdened healthcare systems. The continued challenges of social distancing, containment, isolation, and surge capacity in already stressed hospitals, clinics, and emergency departments have led to a swell in technologically-assisted care delivery strategies, such as telemedicine and web-based triage.

Some of the challenges health care workers are facing right now are; fear of uncertain higher risk of potential infection, worried about being asymptomatic and infecting their loved ones, constant changing recommendations from the government and health leaders, high increase to work longer hours, and balancing helping others while keeping in mind to protect their own mental and physical health. Healthcare workers from around the world are now all facing the high risk of being infected by the virus especially when they are on the front lines testing people for the virus or caring for patients in emergency rooms, and even though they are able to use and put on PPE or personal protective equipment and medical mask are these items are going short in supply in many places. As the race to develop an effective vaccine intensifies, several clinical trials of antivirals and immune modulators are underway, though no reliable COVID-19-specific therapeutics (inclusive of some potentially effective single and multi-drug regimens) have been identified as of yet. With many nations and regions declaring a state of emergency, unprecedented quarantine, social distancing, and border closing efforts are underway [2].

\section{Disparities}

Disparities in health status, access to health care, wealth, employment, wages, housing, income, and poverty all contribute to greater susceptibility to the virus both economically and physically. There are three main groups of workers in the COVID-19 economy: those who have lost their jobs and face economic insecurity, those who are classified as essential workers and face health insecurity as a result, and those who are able to continue working from the safety of their homes [1]. Even though the black and brown communities share many of the experiences that make them more susceptible, there are also important differences between these communities that need to be understood in order to effectively combat the adverse economic and health effects of the virus. This report, focused specifically on black workers, is the first in a series that will explore how racial and economic inequality leave workers of color with few good options for protecting both their health and economic wellbeing. Unfortunately, black workers are less likely to be found in the last group. They have suffered record numbers of job losses over the last two months, along with the ensuing related economic devastation. They also are disproportionately found among the essential workers in the economy today continuing to go to their workplaces, risking their health and that of their families because they are unable to sustain adequate social distance from their co-workers and customers [1]. The global impact of COVID19 , both in lives lost and economic devastation, is likely to leave a lasting mark for years to come. The best path forward includes making sure that we use the painful lessons learned during this crisis to better prepare ourselves for the next one. The disparate racial impact of COVID-19 illustrated in this report should come as no surprise given the ongoing legacy of racism that continues to produce unequal outcomes affecting nearly every aspect of life in the United States. The latest national data available to assess the impact of job losses for black and white workers separately is the Current Population Survey for April 2020. The labor market started deteriorating in March but fell off a cliff in April [1]. While the losses have certainly continued, the April data gives a first look at how black and white workers are faring. The unemployment rate is a commonly used measure of labor market slack. One limitation, however, is that it relies on would-be workers to either be on temporary layoff or have looked for work in the last four weeks to be counted as unemployed. In this economy, with the health requirements to stay home and with sectors being completely decimated, it is likely that many would-be workers are not actively looking for work and therefore would not be counted in the official unemployment rate.

Some of the many inequities health put racial and ethnic minority groups at increased risk of getting sick and dying from COVID-19. Discrimination, which includes racism, can lead to chronic and toxic stress and shapes social and economic factors that put some people from racial and ethnic minority groups at increased risk for COVID-19. Healthcare access can also be limited for these groups by many other factors, such as lack of transportation, child care, or ability to take time off of work; communication and language barriers; cultural differences between patients and providers; and historical and current discrimination in healthcare systems. Some people from racial and ethnic minority groups may hesitate to seek care because they distrust the government and healthcare systems responsible for inequities in treatment and historical events such as the Tuskegee Study of Untreated Syphilis in the African American Male and sterilization without people's permission. Inequities in access to high-quality education for some racial and ethnic minority groups can lead to lower high school completion rates and barriers to college entrance. This may limit future job options and lead to lower paying or less stable jobs. People with limited job options likely have less flexibility to leave jobs that may put them at a higher risk of exposure to the virus that causes COVID-19. People in these situations often cannot afford to miss work, even if they're sick, because they do not have enough money saved up for essential items like food and other important living needs. Some people from racial and ethnic minority groups live in crowded conditions that make it more challenging to follow prevention strategies. In some cultures, it is common for family members of many generations to live in one household. In addition, growing and disproportionate unemployment rates for some racial and ethnic minority groups during the COVID-19 pandemic may lead to greater risk of eviction and homelessness or sharing of housing.

\section{Vaccine}

Vaccine development is a lengthy, expensive process. Attrition is high, and it typically takes multiple candidates and many years to produce a licensed vaccine. Because of the cost and high failure rates, developers typically follow a linear sequence of steps, with multiple pauses for data analysis or manufacturing-process checks. Developing a vaccine quickly requires a new pandemic paradigm with a fast start and many steps executed in parallel before confirming a successful outcome of another step, hence resulting in elevated financial risk. For example, for platforms with experience in humans, phase 1 clinical trials may be able to proceed in parallel with testing in animal models. As soon as China announced that a novel coronavirus had been identified as the cause of the Wuhan outbreak, CEPI (The Coalition for Epidemic Preparedness Innovations) contacted its partners that were developing MERS vaccines or working on novel platforms. With the potential for further financial support, they and others began vaccine development as soon as the first gene sequence was posted, and development is proceeding quickly. It's difficult to predict where and when outbreaks will occur and to prepare trial sites to coincide with vaccine readiness for testing. In addition, if multiple vaccines are ready for testing at the end of 2020, it will be important not to crowd sites or burden countries and their ethics and regulatory authorities with multiple trials, as happened with Ebola therapeutics 
during the 2013-2016 outbreak. Moreover, in a high-mortality situation, populations may not accept randomized, controlled trials with placebo groups; although other approaches that address such concerns may be scientifically feasible, they're typically not as fast, and the results can be harder to interpret [4]. This problem can sometimes be overcome by comparing outcomes with early vaccination versus delayed vaccination. One possible way forward would be to test several vaccines simultaneously in an adaptive trial design using a single, shared control group, so that more participants would receive an active vaccine [1].This approach has advantages but can be logistically and statistically complex, and developers often avoid trials that may generate head-to-head comparative data.

Currently, Pfizer-BioNTech COVID-19 mRNA vaccine is authorized and recommended for use in the United States. Though Moderna's is next, yet AstraZeneca and Janssen are getting ready to introduce their product in the vaccine market for the treatment of COVID-19 disease. The mRNA vaccine technology is not entirely new, very specific, do not alter our DNA, and it triggers an inflammatory response.

\section{Conclusion}

Pandemics propose an immense challenge to public health, heal care systems, and global economic security. Although tremendous progress has been achieved, the continuous evolution of this RNA virus may exert new challenges on the diagnosis and treatment of COVID-19. A detailed study of COVID-19, both genomics and proteomics is needed to know the infection mechanism as well as to drug design. This, however, may be hampered by the lack of an appropriate in vitro as well as in vivo model. Further, future experiments with more sensitive diagnostic tools should yield a more accurate picture of the prevalence of this virus and its association with respiratory diseases. A human vaccine is currently available and is in use to prevent the disease. The highest risk right now, especially for vulnerable people is not to be vaccinated.

\section{References}

1. Economic Policy Institute. (2020) Black workers face two of the most lethal preexisting conditions for coronavirus racism and economic inequality.

2. Center for Disease Control (2019) National Center for Immunization and Respiratory Diseases (NCIRD), Division of Viral Diseases

3. Lu H, Stratton CW and Tang YW (2020) Outbreak of pneumonia of unknown etiology in Wuhan, China: The mystery and the miracle. J Med Virol. 92: 401-402.

4. Velavan TP and Meyer CG. (2020). The Covid-19 epidemic. Trop Med Int Health 25:278-280. 